- Original Article

\title{
The Relationship between Handgrip Strength and Cognitive Function in Elderly Koreans over 8 Years: A Prospective Population-Based Study Using Korean Longitudinal Study of Ageing
}

Kyung Hwan Kim, Soo Kyoung Park, Dong Ryul Lee, Jungun Lee*

Department of Family Medicine, Wonkwang University Sanbon Hospital, Wonkwang University School of Medicine, Gunpo, Korea

Background: Decreased physical performance, such as weakened handgrip strength and cognitive decline, is associated with disability and premature death in old age. We investigated the association between handgrip strength and cognitive impairment in Korean elderly adults with normal cognitive function.

Methods: This prospective study used the database from the Korean Longitudinal Study of Ageing. The participants included 2,378 adults aged 65 years or older with normal cognitive function (Korean Mini-Mental Status Evaluation [K-MMSE] score $\geq 21$ ). Using a mixed-effects model, we examined the associations at baseline and over an 8-year follow-up period between handgrip strength and K-MMSE score. We investigated handgrip strength as a predictor of change in cognitive function.

Results: This study included 1,138 women (mean maximum handgrip strength $19.2 \mathrm{~kg}$, mean K-MMSE score 25.1) and 1,240 men (mean maximum handgrip strength $30.7 \mathrm{~kg}$, mean MMSE score 26.2). The baseline handgrip strength was positively associated with the baseline K-MMSE score $(\beta=0.18, P<0.001)$. Using a mixed-effects model, we found that higher handgrip strength at baseline can predict MMSE scores positively over time $(\beta=0.14, P<0.001)$ and the change of handgrip strength over time was a predictor of high MMSE scores over the study period $(\beta=0.01$, $\mathrm{P}<0.01$ ).

Conclusion: We observed significant associations between baseline handgrip strength and baseline and change of cognition, as well as the longitudinal influence of handgrip strength on the change of cognitive function in elderly Korean adults with normal cognitive function.

Keywords: Cognition; Hand Strength; Aging; Longitudinal Studies

Received: June 14, 2017, Revised: August 10, 2017, Accepted: August 18, 2017

${ }^{*}$ Corresponding Author: Jungun Lee https://orcid.org/0000-0001-8580-1445

Tel: +82-31-390-2416, Fax: +82-31-390-2266, E-mail: tracheo@naver.com 


\section{INTRODUCTION}

Cognitive function decline is observed in middle-aged and young-elderly persons, and is a well-known consequence of the aging process. ${ }^{1,2)}$ With respect to cognition, fluid cognition (the ability to learn new processes and form new memories) has been shown to decline from midlife onwards as a result of normal aging, ${ }^{3,4)}$ whereas cognitive decline of functional or clinical significance (including the diagnosis of dementia or Alzheimer disease) is generally not detected until much later in life. ${ }^{5)}$

Cognitive decline in the elderly has been associated with various factors, such as older age, lower education, lower income, and chronic health conditions including hypertension, stroke, diabetes, and depression. ${ }^{6,7)}$

Age-related decline in physical function is also observed even in the absence of disease from midlife onwards, as a result of normative agerelated changes in the musculoskeletal system and other body systems on which these measures depend. ${ }^{8)}$ Changes in muscle strength may represent age-related changes in biological vitality and physical function. Muscle strength measurements could be a useful part of clinical evaluations in the elderly, to identify persons who have the highest risk for accelerated decline of functional, psychological, and social health in the near future. ${ }^{9)}$

Handgrip strength is often used as a marker not only for muscle strength but also for biological vitality, because it is sensitive to changes related to age and biological functioning. ${ }^{10)}$ Although there is clear evidence of associations between cognition and handgrip strength in terms of performance level, the evidence for longitudinal associations remains unclear. To understand causality, associations should be studied longitudinally. ${ }^{8)}$ To date, only a few longitudinal studies have examined the relationship between handgrip strength and cognition, especially those in which associations with specific cognitive abilities were examined. ${ }^{5,11,12)}$

The objective of this study was to investigate the association between handgrip strength and cognitive ability in elderly Korean adults by using the database of the Korean Longitudinal Study of Ageing (KLoSA) over 8 years. We hypothesized that baseline handgrip strength is associated with baseline cognitive function as measured using the Korean Mini-Mental Status Evaluation (K-MMSE); lower baseline handgrip strength is associated with a higher risk of cognitive decline; and, finally, handgrip strength longitudinally influences the change of cognitive function over an 8-year period.

\section{METHODS}

\section{Data and Study Sample}

This study was based on data from the KLoSA, obtained from baseline interviews from 2006 to the 5th wave in 2014, conducted by the Korea Labor Institute. ${ }^{13)}$ The KLoSA is an ongoing longitudinal panel survey of community-dwelling adults aged $\geq 45$ years. The participants of the KLoSA were selected using multistage stratified probability sampling based on geographical areas. Approximately 10,000 Koreans were interviewed by trained interviewers using computer-assisted personal interviewing methods. The KLoSA survey includes questionnaires on demographics, family, health, employment, income, assets, and subjective expectations and satisfaction. In 2006, the first KLoSA survey was conducted with 10,254 respondents among 6,171 households, and the 5th wave in 2014 comprised 7,029 respondents, representing $72.8 \%$ of the original panel.

This study analyzed the 2006 baseline interview data of 2,378 participants aged 65 years or older with normal cognitive function at the time of the survey. All participants provided written informed consent, and the survey protocol was approved by the Institutional Review Board of Statistics Korea. Among the 10,254 participants of the KLoSA in 2006, 7,399 were excluded because of age ( $<65$ years, $n=6,090$ ), cognitive function (K-MMSE score $<21, \mathrm{n}=1,624$ ), and lack of information ( $n=120)$. Participants who had missing data for handgrip strength ( $\mathrm{n}=148$ ), as well as those with difficulties in daily life caused by cancer, cardiovascular disease, and cerebrovascular disease $(n=209)$ were also excluded. Finally, 2,378 participants (1,240 men and 1,138 women) were analyzed in this study.

This study was reviewed by the Review Board of Sanbon Medical Center of Wonkwang University (IRB approval no., WMCSB 201706$65)$.

\section{Measurement}

The cognitive function of the participants was assessed using the KMMSE, which is a widely used tool for the screening of cognitive impairment in older adults. The validity of the K-MMSE instrument has been established. ${ }^{14)}$ In this study, age, education level, annual household income, body mass index (BMI, $\mathrm{kg} / \mathrm{m}^{2}$ ), smoking status, alcohol consumption, exercise, history of chronic diseases, and depression diagnosis were included as potential covariates. Education level was classified as less than elementary school, middle school, and high school or above college. Additionally, annual household income was classified using gross household income data. BMI was calculated from the body weight and height, and participants were classified into four groups (lean, normal, overweight, or obese) according to the Korean Society for the Study of Obesity. ${ }^{15)}$ The exercise group was defined as those who exercise regularly at least once a week. Chronic diseases were classified as hypertension, diabetes mellitus, chronic lung disease, chronic liver disease, heart disease, and cerebrovascular disease. Alcohol consumption was categorized as current, former, and none. Depression was measured using the 10-item short-form Center for Epidemiological Studies-Depression (CES-D10) scale, a screening tool for assessing depressive conditions. In this study, the Korean edition of CES-D10 for measuring depressive symptoms defined by the American Psychiatric Association's Diagnostic and Statistical Manual 4th edition was used. ${ }^{16)}$ CES-D10 has a score range of 0-10, with a score of $\geq 4$ considered to indicate depression. Activities of daily living (ADL) were measured with the 7-item Korean Activities of Daily Living Scale. ${ }^{17,18)}$ 


\section{Handgrip Strength}

Handgrip strength was measured using a handgrip dynamometer (TANITA Hand Grip Meter Blue 6103; Tanita Co., Tokyo, Japan). The handgrip strengths of the left and right hands were measured twice in a sitting or standing position, and the highest maximum value was used for the study. As respondents were unlikely to be able to perform all four measurements at the same maximum force, only the highest of the four measured values was selected and used in the analysis.

\section{Statistical Analysis}

The baseline characteristics of the study participants were analyzed using Student t-test for continuous variables and the chi-square test for categorical variables. Values are expressed as means \pm standard deviation or as a percentage. Furthermore, baseline associations be- tween handgrip strength and the MMSE score were analyzed using multiple linear regression.

The prospective association between handgrip strength and cognitive decline measured using the K-MMSE was analyzed with a mixedeffects model including estimates for 'handgrip strength,' 'wave,' and 'change of handgrip strength.' The estimate for 'handgrip strength' indicates the baseline association between handgrip strength and the KMMSE score. The estimate for 'wave' indicates the annual change in performance for participants with mean handgrip strength levels. The estimate for 'change of handgrip strength' indicates the accelerated annual decline in handgrip strength from the baseline value. ${ }^{9)}$

A mixed-effects model was chosen for the analysis of the KLoSA data for several reasons. First, the model best accounted for missing or incomplete observations, thus enabling us to use all available informa-

Table 1. Baseline characteristics of study participants $(\mathrm{N}=2,378)$

\begin{tabular}{|c|c|c|c|c|}
\hline Characteristic & Men $(n=1,240)$ & Women $(n=1,138)$ & Total $(\mathrm{N}=2,378)$ & P-value* \\
\hline Age (y) & $71.3 \pm 5.0$ & $71.0 \pm 5.1$ & $71.1 \pm 5.0$ & $<0.01$ \\
\hline Education & & & & $<0.001$ \\
\hline Elementary school or less & $574(46.3)$ & $886(78.0)$ & $1460(61.5)$ & \\
\hline Middle school & $197(15.9)$ & $119(10.5)$ & $316(13.3)$ & \\
\hline High school & $310(25.0)$ & $107(9.4)$ & $417(17.6)$ & \\
\hline University or above & $158(12.8)$ & $24(2.1)$ & $182(7.7)$ & \\
\hline Annual household income (10,000 Korean won) & & & & 0.70 \\
\hline$<1,000$ & $633(51.0)$ & $570(50.1)$ & $1203(50.6)$ & \\
\hline $1,000-2,000$ & $221(17.8)$ & $170(14.9)$ & $391(16.4)$ & \\
\hline $2,000-3,000$ & $134(10.8)$ & $92(8.1)$ & $226(9.5)$ & \\
\hline$\geq 3,000$ & $135(10.9)$ & $134(11.8)$ & $269(11.3)$ & \\
\hline $\mathrm{BMI}\left(\mathrm{kg} / \mathrm{m}^{2}\right)$ & $23.4 \pm 10.2$ & $23.3 \pm 3.6$ & $23.4 \pm 7.8$ & $<0.01$ \\
\hline BMl category ${ }^{\dagger}$ & & & & $<0.001$ \\
\hline Lean & $65(5.3)$ & $71(6.5)$ & $136(5.9)$ & \\
\hline Normal & $572(46.8)$ & $444(40.5)$ & 1016 (43.8) & \\
\hline Overweight & $356(29.1)$ & $299(27.3)$ & $655(28.3)$ & \\
\hline Obese & $230(18.9)$ & $281(25.6)$ & $511(22.0)$ & \\
\hline Smoking status & & & & $<0.001$ \\
\hline Current & $398(32.1)$ & $36(3.2)$ & $434(18.3)$ & \\
\hline Former & $311(25.1)$ & $6(0.5)$ & $317(13.3)$ & \\
\hline None & $531(42.8)$ & $1096(96.3)$ & $1627(68.4)$ & \\
\hline Alcohol consumption & & & & $<0.001$ \\
\hline Current & $198(16.0)$ & $21(1.8)$ & $219(9.2)$ & \\
\hline Former & $693(55.9)$ & $140(12.3)$ & $833(35.0)$ & \\
\hline None & $349(28.1)$ & $977(85.9)$ & 1326 (55.8) & \\
\hline Exercise & $563(45.4)$ & $399(35.1)$ & $962(40.5)$ & $<0.001$ \\
\hline \multicolumn{5}{|l|}{ Chronic disease } \\
\hline Hypertension & $415(33.5)$ & $488(42.9)$ & $903(38.0)$ & $<0.001$ \\
\hline Diabetes mellitus & $189(15.2)$ & $171(15.0)$ & $360(15.1)$ & 0.88 \\
\hline Chronic lung ds. & $58(4.7)$ & $26(2.3)$ & $84(3.5)$ & 0.002 \\
\hline Chronic liver ds. & $28(2.3)$ & $15(1.3)$ & $43(1.8)$ & 0.09 \\
\hline Heart ds. & $39(3.1)$ & $40(3.5)$ & $79(3.3)$ & 0.62 \\
\hline Cerebrovascular ds. & $14(1.1)$ & $11(1.0)$ & $25(1.1)$ & 0.92 \\
\hline Depression & $87(7.0)$ & $165(14.5)$ & $252(10.6)$ & $<0.001$ \\
\hline Handgrip strength (kg) & $30.7 \pm 5.9$ & $19.2 \pm 4.1$ & $25.2 \pm 7.7$ & $<0.01$ \\
\hline Korean-Mini Mental Status Evaluation score & $26.2 \pm 2.4$ & $25.1 \pm 2.5$ & $25.7 \pm 2.5$ & \\
\hline
\end{tabular}

Values are presented as mean \pm standard deviation for continuous variables and number (\%) for categorical variables.

BMl, body mass index; ds., disease.

${ }^{*}$ From Student t-test analysis for continuous variables and from chi-square test for categorical variables. ${ }^{\dagger}$ Category: lean, $<18.5 \mathrm{~kg} / \mathrm{m}^{2}$; normal, $18.5-22.9 \mathrm{~kg} / \mathrm{m}^{2}$; overweight, $23.0-24.9 \mathrm{~kg} / \mathrm{m}^{2}$; obese, $\geq 25.0 \mathrm{~kg} / \mathrm{m}^{2}$. 
tion. Second, it allowed for the modeling of the time-dependent changes in our variables as well as the time-dependent changes in the magnitude of association between the variables. Finally, because the KLoSA data included repeated measures over 8 years, a mixed-effects model allowed us more flexibility in modeling the effects of time on our outcome. ${ }^{6)}$

We also reversed the basic model (direction: handgrip strength to cognition) to test the cognition to handgrip strength direction by using a mixed-effects model. The values of Akaike Information Criteria and Bayesian Information Criteria were used for assessing the model fit, in which lower values indicate a better model fit.

Three mixed-effects model were constructed to test the relationship between handgrip strength and cognitive decline. Model 1 was adjusted for age, sex, and education. In model 2, age, sex, education, BMI, annual household income, smoking status, alcohol consumption, and exercise were adjusted. In model 3, age, sex, education, BMI, annual household income, smoking status, alcohol consumption, exercise, $\mathrm{ADL}$, and chronic disease were adjusted. The data were analyzed using the IBM SPSS statistical package ver. 21.0 for Windows (IBM Corp., Armonk, NY, USA). Values of $\mathrm{P}<0.05$ were considered statistically significant.

\section{RESULTS}

The baseline characteristics of the study participants by sex are presented in Table 1 . The mean age was $71.3 \pm 5.0$ years for male participants and 71.0 \pm 5.1 years for female participants $(\mathrm{P}<0.01)$. Overall, $48.0 \%$ of men and $52.9 \%$ of women were overweight and obese; however, the mean BMI was significantly higher in men than in women $(\mathrm{P}<0.01)$. The smoking and drinking rates of men were significantly higher than those of women $(\mathrm{P}<0.001)$. In history of chronic diseases,

Table 2. Model fit between handgrip strength and cognition

\begin{tabular}{ccc}
\hline \multirow{2}{*}{ Models } & \multicolumn{2}{c}{ Mini-Mental Status Evaluation } \\
\cline { 2 - 3 } & AlC $^{*}$ & BIC $^{*}$ \\
\hline Grip strength to cognition & $40,449.8^{\dagger}$ & $40,477.4^{\dagger}$ \\
Cognition to grip strength & $50,810.5^{\dagger}$ & $50,838.2^{\dagger}$ \\
\hline
\end{tabular}

AIC, Akaike Information Criteria; BIC, Bayesian Information Criteria.

${ }^{*} \mathrm{AIC}$ and BIC were used for assessing model fit. Lower values indicate better model fit. ${ }^{\dagger} \mathrm{P}<0.001$. hypertension (38.0\%) and diabetes mellitus (15.1\%) were the most common medical conditions for both men and women. In this study, $7.0 \%$ of men and $14.5 \%$ of women had a history of depression. The mean handgrip strength of men was higher than that of women $(\mathrm{P}<0.001)$. Thus, the mean K-MMSE score of men was also higher than that of women $(\mathrm{P}<0.01)$.

The association between handgrip strength and cognitive function is shown in Table 2. Comparisons between the two directions, 'handgrip strength to cognition' and 'cognition to handgrip strength,' showed that 'handgrip strength to cognition' had a better model fit according to the criteria. Therefore, we focused on the direction 'handgrip strength to cognition.'

The baseline cross-sectional associations between handgrip strength and the K-MMSE score are shown in Table 3. According to the results in fully adjusted model 3 , the baseline handgrip strength was positively associated with the baseline K-MMSE score $(\beta=0.18$, $\mathrm{P}<0.001)$. The MMSE scores also significantly increased when the education levels $(\beta=0.23, \mathrm{P}<0.001)$ were high. In contrast, the MMSE scores decreased with a statistically significant difference when the age and ADL were high (age: $\beta=-0.12, \mathrm{P}<0.001$; ADL: $\beta=-0.06, \mathrm{P}=0.004$ ).

The relationship between the baseline handgrip strength and changes in handgrip strength and average change in the K-MMSE score over an 8-year follow-up period are presented in Table 4 . In the fully adjusted models, the baseline handgrip strength was a significant predictor of the change of the K-MMSE scores $(\beta=0.14, \mathrm{P}<0.001)$. Furthermore, less decline in handgrip strength was significantly associat-

Table 3. Baseline cross-sectional associations between handgrip strength and Korean-Mini Mental Status Evaluation score

\begin{tabular}{|c|c|c|c|c|c|c|}
\hline \multirow{2}{*}{ Variable } & \multicolumn{2}{|c|}{ Model 1} & \multicolumn{2}{|c|}{ Model 2} & \multicolumn{2}{|c|}{ Model 3} \\
\hline & $\beta$ & P-value* & $\beta$ & P-value* & $\beta$ & P-value* \\
\hline Handgrip strength & 0.19 & $<0.001$ & 0.20 & $<0.001$ & 0.18 & $<0.001$ \\
\hline Age & -0.13 & 0.06 & -0.12 & $<0.001$ & -0.12 & $<0.001$ \\
\hline Sex & 0.01 & 0.99 & 0.01 & 0.87 & -0.01 & 0.81 \\
\hline Education & 0.24 & $<0.001$ & 0.24 & $<0.001$ & 0.23 & $<0.001$ \\
\hline
\end{tabular}

Model 1: adjusted for age, sex, and education; model 2: adjusted for age, sex, education, BMl, annual household income, smoking status, alcohol consumption, and exercise; and model 3: adjusted for age, sex, education, BMI, annual household income, smoking status, alcohol consumption, exercise, activities of daily living, and chronic disease.

$\beta$, regression coefficient, $B M l$, body mass index.

${ }^{\star}$ Analyzed using multiple linear regression.

Table 4. Relationship between baseline K-MMSE score and changes in K-MMSE and average change in handgrip strength over the 8-year follow-up

\begin{tabular}{|c|c|c|c|c|c|c|}
\hline \multirow{2}{*}{ Variable } & \multicolumn{2}{|c|}{ Total } & \multicolumn{2}{|c|}{ Men } & \multicolumn{2}{|c|}{ Women } \\
\hline & $\beta$ (SE) & P-value* & $\beta$ (SE) & P-value* & $\beta$ (SE) & P-value \\
\hline Wave & $-0.51(0.10)$ & $<0.001$ & $-0.12(0.16)$ & 0.45 & $-0.68(0.15)$ & $<0.001$ \\
\hline Baseline grip strength & $0.14(0.01)$ & $<0.001$ & $0.16(0.02)$ & $<0.001$ & $0.17(0.02)$ & $<0.001$ \\
\hline Change of grip strength & $0.01(0.003)$ & $<0.01$ & $-0.001(0.005)$ & 0.86 & $0.01(0.006)$ & 0.05 \\
\hline Intercept & $21.62(1.32)$ & $<0.001$ & $20.86(1.70)$ & $<0.001$ & $20.53(2.19)$ & $<0.001$ \\
\hline
\end{tabular}

K-MMSE, Korean-Mini Mental Status Evaluation; $\beta$, regression coefficient; SE, standard error.

*Analyzed using linear mixed-effects model adjusted for age, sex, education, body mass index, annual household income, smoking status, alcohol consumption, exercise, activities of daily living, and chronic disease. 
ed with less decline in the K-MMSE score over the study period $(\beta=0.01, P<0.01)$. These associations remained significant for handgrip strength: a change of $1 \mathrm{~kg}$ in handgrip strength was associated with a $0.01 \pm 0.003$ decline in the K-MMSE score. In subgroup analysis, we found that higher handgrip strength at baseline can predict the MMSE scores positively over time both in men $(\beta=0.16, P<0.001)$ and women $(\beta=0.17, \mathrm{P}<0.001)$. However, the change of handgrip strength over time was a predictor of high MMSE scores over the study period only in women $(\beta=0.01, P=0.05)$.

\section{DISCUSSION}

From our population-based longitudinal study that monitored handgrip strength and cognition over 8 years, we examined the associations between cognition and handgrip strength in terms of the level of performance and rate of change in the elderly (age $>65$ years). We found significant associations between baseline handgrip strength and baseline cognitive function, and between baseline handgrip strength and change of cognition. Furthermore, we observed the longitudinal influence of handgrip strength on the change of cognitive function.

The results of the present study are consistent with those of previous studies showing that reduced muscle strength and sarcopenia are associated with a risk of cognitive dysfunction. ${ }^{1,2)}$ On the basis of crosssectional studies, we observed a strong association between declines in cognition and physical function. A previous community-based 3.5year follow-up study linked handgrip strength to memory decline in the elderly. ${ }^{19)}$ In addition, another study reported that reduced handgrip strength was associated with an increased risk of Alzheimer disease in healthy elderly persons with mild cognitive impairment (i.e., cognitive impairment level that does not meet the criteria for dementia) ${ }^{20)}$ A recent study in the Korean elderly also reported that handgrip strength was associated with a risk of mild cognitive impairment. ${ }^{1)}$

Only a few longitudinal studies have examined the associations between a simultaneous change in cognition and handgrip strength using multi-wave measurements. ${ }^{10,11,21)}$ Sternäng et al. ${ }^{10)}$ examined change in cognition as a function of age and change in handgrip strength by using time-variant covariant models. They found that change in handgrip strength preceded change in several cognitive domains, such as verbal ability, spatial ability, processing speed, and memory, and that association became more evident after the age of 65 years. Björk et al. ${ }^{21)}$ showed potential associations between change in cognition and change in handgrip strength in relation to impending death in a population-based sample aged $>80$ years at baseline that was followed until death (ОСТО-Twin Study, Origins of Variance in the Old-Old).

On the other hand, Deary et al. ${ }^{11)}$ investigated the trajectories of the changes in the associations between fluid ability and handgrip strength. Participants (79 years of age) were followed up longitudinally with three waves of data on both handgrip strength and reasoning. Cross-sectional relationships between these two variables were found; however, no longitudinal relationships were observed.
There has been one longitudinal study in the Asian population. Auyeung et al. ${ }^{22)}$ examined the association between baseline frailty measurements and cognitive function 4 years later. They showed that physical frailty and weaker handgrip strength both in men and women were associated with cognitive decline over a 4 -year period. However, they measured physical and cognitive function only 2 times rather than using multi-wave measurements. ${ }^{22)}$

In our study, an association between change of handgrip strength and change of MMSE was observed in women but not in men. This may be because the decline of cognition measured using the MMSE score was relatively smaller in men than in women ( -0.12 versus -0.68$)$. To evaluate the meaningful association in men, more long-term follow-up observations would be required.

Although a connection between physical functioning and cognitive abilities has been suggested, the etiology of that relationship has yet to be fully explained. Three main possibilities have been proposed. ${ }^{10}$ Physical functioning drives age-related changes in cognition; ${ }^{6,22)}$ cognition drives age-related changes in physical functioning, ${ }^{23,24)}$ or a third factor affects both. ${ }^{23,25)}$

In comparisons between the two directions 'handgrip strength to cognition' and 'cognition to handgrip strength', our results showed that 'handgrip strength to cognition' had a better model fit for cognitive functions measured using the K-MMSE. Our result is in line with previous research, ${ }^{1)}$ including some studies that reported that handgrip strength predicted cognition. ${ }^{6,22)}$

The proposed mechanisms for the relationship between handgrip strength and cognition in healthy adults involve brain-aging processes, such as the functioning of the central nervous system or changes in white matter integrity. ${ }^{23,25)}$ Another common suggestion about the causal role of aging is that the aging process operates at various levels, from brain circuitry ${ }^{26)}$ and brain pathology ${ }^{27)}$ to loss of molecular fidelity. $^{28)}$

Many studies have shown that cognitive decline in the elderly is associated with various factors, such as older age and lower education and income levels, and a high level of muscle strength is associated with chronic health conditions including hypertension, stroke, diabetes, and depression. ${ }^{6,7)}$ In this study, cognitive function was significantly increased in participants with high education levels and those with a history of diabetes mellitus.

The present study has some strengths that should be highlighted, such as its large community sample size, the prospective design (8year follow-up period), ${ }^{6)}$ concurrent cognitive and physical assessments using validated measures, and availability of a number of lifestyle and health variables. Thus, the current findings can be generalized to the entire Korean population. Second, previous longitudinal studies on handgrip strength and cognitive function were limited to the Caucasian and Hispanic ethnicities; this is the first multi-wave longitudinal study in participants of Asian ethnicity.

However, the study also has a few limitations that need to be acknowledged. First, we used single measures of cognition (K-MMSE) and physical function (handgrip strength). According to life span, the 
domain of cognitive impairment does not develop simultaneously. Björk et al. ${ }^{21)}$ showed that cognition and handgrip strength in later life differ across specific domains, rather than being indicators of general cognitive performance. Thus, future studies should incorporate a wider range of physical and cognitive measures. Second, although we attempted to overcome this problem by using a mixed-effects model, missing data might have influenced the results during the follow-up period. Third, our data were limited to self-reports of medical conditions and we did not use a biomarker that could explain a common mechanism shared by cognitive decline and muscle loss in relation to age.

Nonetheless, to our knowledge, this is the first study to show the longitudinal influence of handgrip strength on the change of cognition measured using the MMSE score. Future studies with a broader range of cognition tasks and other physical functions are needed to better describe the developmental associations between cognition and muscle function, and other mediators should be investigated to evaluate the common mechanism of the decline of both cognition and physical function.

\section{CONFLICT OF INTEREST}

No potential conflict of interest relevant to this article was reported.

\section{ACKNOWLEDGMENTS}

This paper was supported by Wonkwang University in 2017.

\section{REFERENCES}

1. Jang JY, Kim J. Association between handgrip strength and cognitive impairment in elderly Koreans: a population-based cross-sectional study. J Phys Ther Sci 2015;27:3911-5.

2. Anstey KJ, Smith GA. Interrelationships among biological markers of aging, health, activity, acculturation, and cognitive performance in late adulthood. Psychol Aging 1999;14:605-18.

3. Singh-Manoux A, Kivimaki M, Glymour MM, Elbaz A, Berr C, Ebmeier KP, et al. Timing of onset of cognitive decline: results from Whitehall II prospective cohort study. BMJ 2012;344:d7622.

4. Richards M, Shipley B, Fuhrer R, Wadsworth ME. Cognitive ability in childhood and cognitive decline in mid-life: longitudinal birth cohort study. BMJ 2004;328:552.

5. Clouston SA, Brewster P, Kuh D, Richards M, Cooper R, Hardy R, et al. The dynamic relationship between physical function and cognition in longitudinal aging cohorts. Epidemiol Rev 2013;35:33-50.

6. Alfaro-Acha A, Al Snih S, Raji MA, Kuo YF, Markides KS, Ottenbacher KJ. Handgrip strength and cognitive decline in older Mexican Americans. J Gerontol A Biol Sci Med Sci 2006;61:859-65.

7. Guadalupe-Grau A, Carnicero JA, Gomez-Cabello A, Gutierrez Avila G, Humanes S, Alegre LM, et al. Association of regional muscle strength with mortality and hospitalisation in older people. Age Ageing 2015;44:790-5.

8. Cooper R, Hardy R, Aihie Sayer A, Ben-Shlomo Y, Birnie K, Cooper C, et al. Age and gender differences in physical capability levels from mid-life onwards: the harmonisation and meta-analysis of data from eight UK cohort studies. PLoS One 2011;6:e27899.

9. Taekema DG, Gussekloo J, Maier AB, Westendorp RG, de Craen AJ. Handgrip strength as a predictor of functional, psychological and social health: a prospective population-based study among the oldest old. Age Ageing 2010;39:331-7.

10. Sternang O, Reynolds CA, Finkel D, Ernsth-Bravell M, Pedersen NL, Dahl Aslan AK. Grip strength and cognitive abilities: associations in old age. J Gerontol B Psychol Sci Soc Sci 2016;71:841-8.

11. Deary IJ, Johnson W, Gow AJ, Pattie A, Brett CE, Bates TC, et al. Losing one's grip: a bivariate growth curve model of grip strength and nonverbal reasoning from age 79 to 87 years in the Lothian Birth Cohort 1921. J Gerontol B Psychol Sci Soc Sci 2011;66:699-707.

12. Kuh D, Cooper R, Hardy R, Guralnik J, Richards M; Musculoskeletal Study Team. Lifetime cognitive performance is associated with midlife physical performance in a prospective national birth cohort study. Psychosom Med 2009;71:38-48.

13. Jang SN. Korean Longitudinal Study of Ageing (KLoSA): overview of research design and contents. In: Pachana N, editor. Encyclopedia of geropsychology. Singapore: Springer; 2016.

14. Kang Y, Na DL, Hahn S. A validity study on the Korean Mini-Mental State Examination (K-MMSE) in dementia patients. J Korean Neurol Assoc 1997;15:300-8.

15. Kim SM, Lee DJ, Kim YS, Lee TH. Assessment of anthropometric indices of obesity in Korea. J Korean Soc Study Obes 2000;9:267-77.

16. Prisciandaro JJ, Roberts JE. A comparison of the predictive abilities of dimensional and categorical models of unipolar depression in the National Comorbidity Survey. Psychol Med 2009;39:1087-96.

17. Lee SH, Kim YB. Which type of social activities decrease depression in the elderly?: an analysis of a population-based study in South Korea. Iran J Public Health 2014;43:903-12.

18. Jeon GS, Jang SN, Kim DS, Cho SI. Widowhood and depressive symptoms among Korean elders: the role of social ties. J Gerontol B Psychol Sci Soc Sci 2013;68:963-73.

19. Christensen H, Mackinnon AJ, Korten AE, Jorm AF, Henderson AS, Jacomb P, et al. An analysis of diversity in the cognitive performance of elderly community dwellers: individual differences in change scores as a function of age. Psychol Aging 1999;14:365-79.

20. Boyle PA, Buchman AS, Wilson RS, Leurgans SE, Bennett DA. Association of muscle strength with the risk of Alzheimer disease and the rate of cognitive decline in community-dwelling older persons. Arch Neurol 2009;66:1339-44.

21. Bjork MP, Johansson B, Hassing LB. I forgot when I lost my grip-strong associations between cognition and grip strength in level of performance and change across time in relation to impending death. Neurobiol Aging 2016;38:68-72.

22. Auyeung TW, Lee JS, Kwok T, Woo J. Physical frailty predicts future cognitive decline: a four-year prospective study in 2737 cognitively normal older adults. J Nutr Health Aging 2011;15:690-4.

23. Christensen H, Mackinnon AJ, Korten A, Jorm AF. The "common cause hypothesis" of cognitive aging: evidence for not only a common factor but also specific associations of age with vision and grip strength in a cross-sectional analysis. Psychol Aging 2001;16:588-99.

24. Charles LE, Burchfiel CM, Fekedulegn D, Kashon ML, Ross GW, Sand- 
erson WT, et al. Occupational and other risk factors for hand-grip strength: the Honolulu-Asia Aging Study. Occup Environ Med 2006;63:820-7.

25. Baltes PB, Lindenberger U. Emergence of a powerful connection between sensory and cognitive functions across the adult life span: a new window to the study of cognitive aging? Psychol Aging 1997;12: 12-21.

26. Baillieux H, de Smet HJ, Paquier PF, de Deyn PP, Marien P. Cerebellar neurocognition: insights into the bottom of the brain. Clin Neurol
Neurosurg 2008;110:763-73.

27. Penke L, Munoz Maniega S, Murray C, Gow AJ, Hernandez MC, Clayden JD, et al. A general factor of brain white matter integrity predicts information processing speed in healthy older people. J Neurosci 2010;30:7569-74.

28. Farooqui T, Farooqui AA. Aging: an important factor for the pathogenesis of neurodegenerative diseases. Mech Ageing Dev 2009;130:20315. 